\title{
Convalescent plasma therapy as a conventional trick for treating COVID-19: a systematic review and meta-analysis study
}

\author{
Masoud Keikha \\ Mashhad University of Medical Sciences \\ Mohsen Karbalaei ( $\Delta$ mohsen.karbalaei@jmu.ac.ir) \\ Jiroft University of Medical Sciences https://orcid.org/0000-0001-9899-2885
}

\section{Research}

Keywords: SARS-CoV-1, MERS-CoV, SARS-CoV-2, Convalescent plasma, Meta-analysis

Posted Date: November 18th, 2020

DOl: https://doi.org/10.21203/rs.3.rs-110194/v1

License: (c) (1) This work is licensed under a Creative Commons Attribution 4.0 International License.

Read Full License

Version of Record: A version of this preprint was published at New Microbes and New Infections on July 1st, 2021. See the published version at https://doi.org/10.1016/j.nmni.2021.100901. 


\section{Abstract}

Background: Convalescent plasma therapy (CPT) is one of the well-known therapeutic protocols for treating infectious diseases that do not have special treatment nor vaccine. In the CPT procedure, the plasma of patients which has the high titers of neutralizing antibodies is transfused to other infected patients who have been affected by the same infectious agent. Several documents confirm the clinical efficacy of this therapy for treating bacterial and viral infections.

Methods: The comprehensive systematic search was conducted in several databases including PubMed, Scopus, Embase, Cochrane library, Google scholar, medRxiv, and bioRxiv. The Joanna Briggs Institute (JBI) critical appraisal checklist was used for the evaluation of included studies. Using the Comprehensive Meta-Analysis (CMA) software version 2.2 (Biostat, Englewood, NJ, USA), the pooled data analysis was performed.

Results: The statistical analysis showed that clinical improvement in the group of patients who received $\mathrm{CP}$ significantly increased compared to the control group. (OR: $2.23 ; 1.12-4.45$ with $95 \% \mathrm{Cls} ; p$ value: 0.022; $Q$-value: 6.11 ; $P$ : 83.64; Eggers $p$ value: 0.064 ; Beggs $p$ value: 0.093$)$. Furthermore, discharge rate of patients from hospital in the group that were received CPT had increased (OR: 2.92; 1.48-5.77 with 95\% Cls; $p$ value: 0.002 ; $Q$-Value: 4.32 ; 2 : 53.80 ; Eggers $p$ value: 0.32 ; Beggs $p$ value: 0.50 ).

Conclusions: Regarding the lack of a specific vaccine, we need to use alternative methods to complete the clinical trials. CPT is among the well-known therapeutic protocols that its clinical trials in treating the coronaviruses infections have been confirmed.

\section{Introduction}

The family Coronaviridae is known as one of the most important etiologic factors for severe acute respiratory diseases for 21 st-century human beings. The members of this family are enveloped, singlestranded, positive-sense RNA viruses (26-32 kbp length), and also have many spikes proteins on their surfaces that mediate virus entry into the cells (1). Coronaviruses have a broad spectrum of the host including avian, humans, and several mammals such as bats, camels, mice, cats, dogs, and anteaters (2). Among them, several coronaviruses (CoVs) such as 229E, OC43, NL63, HKU1, SARS-CoV-1, MERS-CoV, and more recently severe acute respiratory syndrome coronavirus 2 (SARS-CoV-2) can infect human (3). Before 2002, CoVs were known only as of the causes of common cold, however, in the 21 st century, the large pandemic of betacoronavirus revealed that these RNA viruses can cause the life-threatening severe respiratory diseases (4). In 2002, the first pandemic, severe acute respiratory syndrome (SARS), was caused by SARS-CoV-1, so that had the mortality rate about $10 \%$ (5). The second pandemic, Middle East respiratory syndrome (MERS), was occurred by MERS-CoV in 2012, with mortality rate about $35 \%$ (6). In December 2019, a new member of betacoronavirus, 2019 novel coronavirus (2019-nCoV) emerged in Wuhan, China, causing a severe pneumonia, that was called coronavirus disease 2019 (COVID-19) (7). The clinical manifestations of COVID-19 are fever, dyspnea, myalgia, and invasive multi-lobular lesions 
(in chest radiological findings), and these are much like SARS and MERS diseases $(4,8)$. The nucleotide sequence of the 2019-nCoV genome is very similar (88\%) with both bat-SL-CoVZC45 and bat-SLCoVZXC21 genomes. In addition, the phylogenetic analysis revealed that the similarity of its genome with both SARS-CoV-1 and MERS-CoV is $79 \%$ and $50 \%$ respectively (9). SARS-CoV-2 is a highly contagious virus, and rapidly spread worldwide. In March 2020, World Health Organization (WHO) announced the COVID-19 pandemic, and nowadays more than 13 million cases are infected by this virus (10). Despite the rapid spread of the virus, but so far there is no an approved vaccine or drug approved by Food and Drug Administration (FDA) against COVID-19 (11). The lack of a protective vaccine and yet the need to avoid of spread of virus has been led to use of alternative strategies such as convalescent plasma therapy (CPT) for the treatment of patients (12). Historically, passive immunization is as one the therapeutic protocols against infectious agents, and first was used in 1880s (13). In passive immunization, the individuals who recover from an infectious disease are investigated, and the convalescent plasma (CP) with high titers of neutralizing antibodies is used for other similar patients, so that leads to reduce the clinical sings, treatment duration and mortality rate (14). According to review of literature, CP has been used for treating diseases such as Diphtheria, Spanish influenza, Ebola, West Nile fever, SARS, and MERS, and has been satisfactory results in amelioration and reduction of mortality rate (15-19). Due to advantages such clinical efficacy, viral therapy, reducing death, and low side effects, CPT is considered as a suitable therapeutic option in complications such infectious diseases, immune deficiencies, allergies, and autoimmune diseases $(13,20,21)$. Furthermore, based on studies, it is demonstrated that CP has the satisfying results in improving and increasing the survival of COVID-19 patients (22). CPT is one of the most reliable therapeutic options during the outbreaks of infectious agents, in particular in the absence of the appropriate vaccine (12). Recently, FDA has announced that CP can be used as a trustworthy way in cases of widespread outbreak of COVID-19 (23). In the present metaanalysis, we fulfilled a comprehensive evaluation study about the effects of CP on clinical improvement, increase of discharged cases, as well as reducing mortality of infected patients by viruses SARS-CoV-1, MERS-CoV, and SARS-CoV-2.

\section{Methods}

\subsection{Search strategy}

The comprehensive systematic search was conducted in several databases including PubMed, Scopus, Embase, Cochrane library, Google scholar, medRxiv, and bioRxiv. Our search strategy was based on MeSH and using keywords such as "convalescent plasma", "COVID-19", "SARS-CoV-1", "MERS-CoV", "SARS-CoV2 ", and "Coronavirus". Next, we retrieved all relevant articles (up to August 2020) about the evaluation of $\mathrm{CP}$ effect on infected patients by SARS-CoV-1, MERS-CoV, and SARS-CoV-2. The inclusion criteria were included: 1) articles containing the characteristics such as clinical improvement, viral therapy, mortality rate, the number of discharged cases, and adverse event rate, in CP therapy patients; 3) patients infected by SARS-CoV-1, MERS-CoV, and SARS-CoV-2; 3) articles containing the full text; 4) English articles. Also, duplicate studies were considered as exclusion criteria (Fig. 1).

\subsection{Quality assessment and data extraction}

Page $3 / 11$ 
The Joanna Briggs Institute (JBI) critical appraisal checklist was used for the evaluation of included studies. The main findings and characteristics of included studies include first author, country, viral etiology, number of patients, CP dosages, outcome endpoint, number of improved patients, number of death, viral therapy rate, adverse event rate, number of cases weaned from the mechanical ventilation, number of discharged cases, therapeutic received drugs, and reference number. The information is summarized in Table 1.

\subsection{Quantitative synthesis}

Using the Comprehensive Meta-Analysis (CMA) software version 2.2 (Biostat, Englewood, NJ, USA), the pooled data analysis was performed. In the present study, the cases who had received CP therapy were evaluated from aspects such as clinical improvement, discharged from hospital, weaned from mechanical ventilation, viral therapy, adverse events, and mortality rate. For assessing the mentioned information we used from event rate with $95 \%$ confidence intervals (Cls). Also, for evaluating the clinical efficacy of CP therapy was used from the Odds Ratio (OR) with $95 \% \mathrm{Cls}$ as well. Using the random-effects model, the pooled OR was estimated. It is noteworthy that, based on the Dersimonian and Laird method and random-effects model, the high heterogeneity cases included $P$ index $>25 \%$ and Cochrane $Q$ test $p$ value $\leq 0.05$

\section{Results}

In the present meta-analysis, from all fifteen studies (5,240 participants) eight studies were on SARS-CoV, while the other two and five were about MERS-CoV and SARS-CoV-1 respectively. The studies had been conducted in four countries China, Korea, Taiwan, and the United States (USA). From all of the infected patients, 5151 were infected by SARS-CoV-2, while four patients were infected by MERS-CoV, as well as eighty-five were infected by SARS-CoV-1. The patients had received $200-900 \mathrm{~mL}$ of $\mathrm{CP}$ in addition to medication with steroids, antibiotics, anti-fungi drugs, and also anti-viral drugs containing lopinavir/ritonavir, favipiravir, IFN-alpha 1b, arbidol, darunavir, ribavirin, remdesivir, and peramivir. However, the parameters such as IgG titer, therapeutic regimens, disease status, and outcome endpoint varied in different patients.

Overall, the results indicate that the CPT has good clinical effects on the patients infected by CoVs (SARS-CoV-1, MERS-CoV, and SARS-CoV-2). Except for antiviral effects, the recovery rate in patients that had received CP was satisfying; adverse events or even mortality rate was low as well. The statistical analysis showed that clinical improvement in the group of patients who received CP significantly increased compared to the control group. (OR: $2.23 ; 1.12-4.45$ with $95 \% \mathrm{Cls} ; p$ value: $0.022 ; Q$-value: 6.11 ; P: 83.64; Eggers $p$ value: 0.064 ; Beggs $p$ value: 0.093$)$. Furthermore, discharge rate of patients from hospital in the group that were received CPT had increased (OR: 2.92; 1.48-5.77 with 95\% Cls; $p$ value: 0.002 ; $Q$-Value: 4.32; 1 : 53.80 ; Eggers $p$ value: 0.32 ; Beggs $p$ value: 0.50 ). In the sub-grouping analysis, we determined the efficacy of CPT in each patient separately. The statistical analysis on the eighty-five patients infected by SARS-CoV-1 demonstrated that the parameters such as clinical improvement, weaning from mechanical ventilation, and discharging from the hospital in patients under the CPT 
significantly had increased (OR: $2.477 ; 0.984-3.97$ with $95 \%$ Cls; $p$ value: $0.001 ; Q$-Value: $0.00 ; I^{2}: 0.00 ; p$ value: 1.00 ). On the other hand, the rate of mortality had a significant decrease in patients who had received CP compared to the control group (OR: 0.077 ; $0.004-1.497$ with $95 \%$ Cls; $p$ value: $0.090 ; Q$-Value: $0.00 ; P: 0.00 ; p$ value: 1.00 ). In the group infected by MERS-CoV, we analyzed the information of four patients. Although due to the low sample size we didn't achieve significant results, however, using CPT had satisfying results in improving the clinical manifestations. Interestingly, statistical analysis of information of 5,151 COVID-19 patients confirmed the efficacy of CPT during the treatment. Improvement of clinical symptoms in patients who had received $\mathrm{CP}$ was more compared to the control group, although we didn't observe the significant changes (OR: $1.66 ; 0.78-3.53$ with $95 \%$ Cls; $p$ value: 0.183 ; $Q$-Value: 2.613; $1:$ : 61.72; $p$ value: 0.106 ). Negative virus test for this disease in recipients of $C P$ had the meaningful change (OR: $2.59 ; 1.65-3.52$ with $95 \%$ Cls; $p$ value: $0.001 ; Q$-Value: $0.67 ; 12: 0.00 ; p$ value: 0.41 ). To discharge the patients in this group significantly increased as well (OR: 2.02; $0.94-4.35$ with $95 \% \mathrm{Cls} ; p$ value: $0.07 ; Q$-Value: $0.049 ; P^{2}: 0.00 ; p$ value: 0.824$)$. As well as, based on statistical analysis results, $\mathrm{CP}$ could be significantly decreased the mortality rate in COVID-19 patients (OR: $0.311 ; 0.12-0.76$ with $95 \%$ Cls; p-Value: 0.011; Q-Value: 0.009; P: 0.00; p-Value: 0.923). The mentioned data are listed in Table 2.

\section{Discussion}

So far, several genera of family Coronaviridae such as 229E, OC43, NL63, HKU1, SARS-CoV-1, and MERSCoV have been known, however, in 2019, a new genus of this family, SARS-CoV-2, was considered as the etiologic agent of COVID-19 (38). The first outbreak of COVID-19 occurred in more than 800 health-care workers in Wuhan, China, however, the disease rapidly was spread in other countries, in particular Thailand, Japan, South Korea, and the USA $(4,39,40)$. At the moment several drugs such as teicoplanin, hydroxychloroquine, remdesivir, lopinavir, oseltamivir, ribavirin, favipiravir are used for the treatment of COVID-19 (9). Currently, regarding the importance of COVID-19 on one hand, and lack of the protective vaccine or effective drug, on the other hand, COVID-19 convalescent plasma (CCP) therapy can be considered as one of the fundamental ways for the treatment of this disease (41). Studies show that the transfusion of plasma from patients who have recovered from COVID-19 infection to other SARS-CoV-2infected patients can be led to their treatment without the occurrence of severe adverse events (42). According to a study that Shen et al. conducted on five critically ill patients, following plasma transfusion, body temperature normalized during 3 days in four patients, the viral load became negative within two weeks after CCP therapy in all patients, and also, and 3 patients were weaned from mechanical ventilation (24). Ye et al. conducted a study on six COVID-19 patients who had been admitted to Wuhan Huoshenshan Hospital from 11th February to 12th March 2020, and among them, there was a patient with Sjögren syndrome as well. They observed that the treatment of patients with CP had satisfying outcomes in all of them. Although the exact mechanism of action of CPT is not understood, they proposed that the antibodies IgM and IgG can directly neutralize the SARS-CoV-2, and probably, the antiinflammatory contents of CP prevent cytokine storms (28). In another study, Ahn et al. estimated the viral load of two cases that were affected by COVID-19 and acute respiratory distress syndrome (ARDS) by rRT-PCR technique, before and after CPT. In both cases, the value of the cycle threshold (Ct) had changed, 
which indicated a reduction in viral load after CP transfusion. In case $1 \mathrm{Ct}$ changed from 24.98 on day 10 to 33.96 on day 20 after plasma transfusion, and in case $2 \mathrm{Ct}$ changed from 20.51 on day 5 to 36.33 on day 9 after plasma transfusion (27). Li et al. (2020) realized that CPT could be led to the negative conversion of SARS-CoV-2 in PCR (OR: 11.39; 3.91-33.18 with 95\% Cls; p-Value: 0.01) (22). Based on studies, the exception with itching or skin rash and sometimes the little increase in body temperature, so far no serious adverse events have been reported with CPT (43).

\section{Conclusion}

Based on different studies, it seems that CPT is an appropriate alternative for the treatment of infected patients by CoVs. The results of the present meta-analysis represented the clinical efficacy of CPT against CoVs. In this study, we showed that $\mathrm{CP}$ could be a candidate for treating the patients who were infected by CoVs. This therapeutic protocol is an effect on characteristics such as clinical improvement, weaning from mechanical ventilation, hospital discharge, viral treatment, and also prevention of mortality.

\section{Abbreviations}

Coronaviruses (CoVs)

Severe acute respiratory syndrome coronavirus 2 (SARS-CoV-2)

Middle East respiratory syndrome (MERS)

2019 novel coronavirus (2019-nCoV)

Coronavirus disease 2019 (COVID-19)

World Health Organization (WHO)

Food and Drug Administration (FDA)

Convalescent plasma therapy (CPT)

Convalescent plasma (CP)

Joanna Briggs Institute (JBI)

Comprehensive Meta-Analysis (CMA)

Confidence intervals (Cls)

Odds Ratio (OR)

United States (USA) 
Acute respiratory distress syndrome (ARDS)

\section{Declarations}

Ethics approval and consent to participate

Not applicable (this paper was provided based on research in global databases)

Consent to publish

Not Applicable

\section{Availability of data and materials}

All data generated or analyzed during this study are included in this published article and its supplementary information files

\section{Competing interests}

There is no conflict of interest among all authors.

\section{Funding}

We have not received any funding for this research.

\section{Authors' Contributions}

1. MK1 has contributed to the design of the work and analysis of data

2. MK2 have drafted the work and substantively revised it

All authors read and approved the final manuscript

\section{Acknowledgements}

We appreciate both Mashhad University of Medical Sciences and Jiroft University of Medical Sciences.

\section{References}

1. Su S, Wong G, Shi W, Liu J, Lai AC, Zhou J, et al. Epidemiology, genetic recombination, and pathogenesis of coronaviruses. Trends in microbiology. 2016;24(6):490-502.

2. Singhal T. A review of coronavirus disease-2019 (COVID-19). The Indian Journal of Pediatrics. 2020:1-6.

3. Fehr AR, Perlman S. Coronaviruses: an overview of their replication and pathogenesis. Coronaviruses: Springer; 2015. p. 1-23. 
4. Huang C, Wang Y, Li X, Ren L, Zhao J, Hu Y, et al. Clinical features of patients infected with 2019 novel coronavirus in Wuhan, China. The lancet. 2020;395(10223):497-506.

5. Lee N, Hui D, Wu A, Chan P, Cameron P, Joynt GM, et al. A major outbreak of severe acute respiratory syndrome in Hong Kong. New England Journal of Medicine. 2003;348(20):1986-94.

6. de Groot RJ, Baker SC, Baric RS, Brown CS, Drosten C, Enjuanes L, et al. Commentary: Middle east respiratory syndrome coronavirus (mers-cov): announcement of the coronavirus study group. Journal of virology. 2013;87(14):7790-2.

7. Zhu N, Zhang D, Wang W, Li X, Yang B, Song J, et al. A novel coronavirus from patients with pneumonia in China, 2019. New England Journal of Medicine. 2020.

8. Chan JF-W, Yuan S, Kok K-H, To KK-W, Chu H, Yang J, et al. A familial cluster of pneumonia associated with the 2019 novel coronavirus indicating person-to-person transmission: a study of a family cluster. The Lancet. 2020;395(10223):514-23.

9. Yousefi B, Valizadeh S, Ghaffari H, Vahedi A, Karbalaei M, Eslami M. A global treatments for coronaviruses including COVID-19. Journal of Cellular Physiology. 2020.

10. Wilder-Smith A, Chiew CJ, Lee VJ. Can we contain the COVID-19 outbreak with the same measures as for SARS? The Lancet Infectious Diseases. 2020.

11. Rojas M, Rodríguez Y, Monsalve DM, Acosta-Ampudia Y, Camacho B, Gallo JE, et al. Convalescent plasma in Covid-19: Possible mechanisms of action. Autoimmunity Reviews. 2020:102554.

12. Marano G, Vaglio S, Pupella S, Facco G, Catalano L, Liumbruno GM, et al. Convalescent plasma: new evidence for an old therapeutic tool? Blood Transfusion. 2016;14(2):152.

13. Shahani L, Singh S, Khardori NM. Immunotherapy in clinical medicine: historical perspective and current status. Medical Clinics. 2012;96(3):421-31.

14. Casadevall A, Pirofski L-a. The convalescent sera option for containing COVID-19. The Journal of clinical investigation. 2020;130(4):1545-8.

15. Luke TC, Hoffman SL. Blood Products for Spanish Influenza: A Future H5N1 Treatment? Annals of Internal Medicine. 2007;146(9):687.

16. Mair-Jenkins J, Saavedra-Campos M, Baillie JK, Cleary P, Khaw F-M, Lim WS, et al. The effectiveness of convalescent plasma and hyperimmune immunoglobulin for the treatment of severe acute respiratory infections of viral etiology: a systematic review and exploratory meta-analysis. The Journal of infectious diseases. 2015;211(1):80-90.

17. Rojas M, Monsalve DM, Pacheco Y, Acosta-Ampudia Y, Ramírez-Santana C, Ansari AA, et al. Ebola virus disease: An emerging and re-emerging viral threat. Journal of autoimmunity. 2020;106:102375.

18. Planitzer CB, Modrof J, Kreil TR. West Nile virus neutralization by US plasma-derived immunoglobulin products. The Journal of infectious diseases. 2007;196(3):435-40.

19. Jean S-S, Lee P-I, Hsueh P-R. Treatment options for COVID-19: The reality and challenges. Journal of Microbiology, Immunology and Infection. 2020. 
20. Sherer Y, Levy Y, Shoenfeld Y. IVIG in autoimmunity and cancer-efficacy versus safety. Expert opinion on drug safety. 2002;1(2):153-8.

21. Katz U, Achiron A, Sherer Y, Shoenfeld Y. Safety of intravenous immunoglobulin (IVIG) therapy. Autoimmunity reviews. 2007;6(4):257-9.

22. Li L, Zhang W, Hu Y, Tong X, Zheng S, Yang J, et al. Effect of Convalescent Plasma Therapy on Time to Clinical Improvement in Patients With Severe and Life-threatening COVID-19: A Randomized Clinical Trial. Jama. 2020.

23. Food U. Recommendations for investigational COVID-19 convalescent plasma. 2020.

24. Shen C, Wang Z, Zhao F, Yang Y, Li J, Yuan J, et al. Treatment of 5 critically ill patients with COVID-19 with convalescent plasma. Jama. 2020;323(16):1582-9.

25. Joyner MJ, Wright RS, Fairweather D, Senefeld JW, Bruno KA, Klassen SA, et al. Early safety indicators of COVID-19 convalescent plasma in 5,000 patients. The Journal of Clinical Investigation. 2020.

26. Zeng Q-L, Yu Z-J, Gou J-J, Li G-M, Ma S-H, Zhang G-F, et al. Effect of convalescent plasma therapy on viral shedding and survival in patients with coronavirus disease 2019. The Journal of infectious diseases. 2020;222(1):38-43.

27. Ahn JY, Sohn Y, Lee SH, Cho Y, Hyun JH, Baek YJ, et al. Use of convalescent plasma therapy in two COVID-19 patients with acute respiratory distress syndrome in Korea. Journal of Korean medical science. 2020;35(14).

28. Ye M, Fu D, Ren Y, Wang F, Wang D, Zhang F, et al. Treatment with convalescent plasma for COVID-19 patients in Wuhan, China. Journal of Medical Virology. 2020.

29. Zhang B, Liu S, Tan T, Huang W, Dong Y, Chen L, et al. Treatment with convalescent plasma for critically ill patients with SARS-CoV-2 infection. Chest. 2020.

30. Duan K, Liu B, Li C, Zhang H, Yu T, Qu J, et al. Effectiveness of convalescent plasma therapy in severe COVID-19 patients. Proceedings of the National Academy of Sciences. 2020;117(17):9490-6.

31. Ko J-H, Seok H, Cho SY, Ha YE, Baek JY, Kim SH, et al. Challenges of convalescent plasma infusion therapy in Middle East respiratory coronavirus infection: a single centre experience. Antivir Ther. 2018;23(7):617-22.

32. Chun S, Chung CR, Ha YE, Han TH, Ki CS, Kang ES, et al. Possible Transfusion-Related Acute Lung Injury Following Convalescent Plasma Transfusion in a Patient With Middle East Respiratory Syndrome. Annals of laboratory medicine. 2016;36(4):393-5.

33. Wong VW, Dai D, Wu AK, Sung JJ. Treatment of severe acute respiratory syndrome with convalescent plasma. Hong Kong Med J. 2003;9(3):199-201.

34. Yeh K-M, Chiueh T-S, Siu LK, Lin J-C, Chan PKS, Peng M-Y, et al. Experience of using convalescent plasma for severe acute respiratory syndrome among healthcare workers in a Taiwan hospital. The Journal of antimicrobial chemotherapy. 2005;56(5):919-22. 
35. Soo YOY, Cheng Y, Wong R, Hui DS, Lee CK, Tsang KKS, et al. Retrospective comparison of convalescent plasma with continuing high-dose methylprednisolone treatment in SARS patients. Clinical microbiology and infection : the official publication of the European Society of Clinical Microbiology and Infectious Diseases. 2004;10(7):676-8.

36. Kong L. Severe acute respiratory syndrome (SARS). Transfus Apher Sci. 2003;29(1):101.

37. Cheng Y, Wong R, Soo YOY, Wong WS, Lee CK, Ng MHL, et al. Use of convalescent plasma therapy in SARS patients in Hong Kong. European journal of clinical microbiology \& infectious diseases : official publication of the European Society of Clinical Microbiology. 2005;24(1):44-6.

38. Decaro N, Lorusso A. Novel human coronavirus (SARS-CoV-2): A lesson from animal coronaviruses. Veterinary Microbiology. 2020:108693.

39. Wang C, Horby PW, Hayden FG, Gao GF. A novel coronavirus outbreak of global health concern. The Lancet. 2020;395(10223):470-3.

40. Haynes B, Messonnier NE, Cetron MS. First travel-related case of 2019 novel coronavirus detected in United States: press release, Tuesday, January 21, 2020. 2020.

41. Xia X, Li K, Wu L, Wang Z, Zhu M, Huang B, et al. Improved clinical symptoms and mortality on severe/critical COVID-19 patients utilizing convalescent plasma transfusion. Blood. 2020.

42. Chen L, Xiong J, Bao L, Shi Y. Convalescent plasma as a potential therapy for COVID-19. The Lancet Infectious Diseases. 2020;20(4):398-400.

43. Tiberghien P, de Lamballerie X, Morel P, Gallian P, Lacombe K, Yazdanpanah Y. Collecting and evaluating convalescent plasma for COVID-19 treatment: why and how? Vox sanguinis. 2020.

\section{Figures}




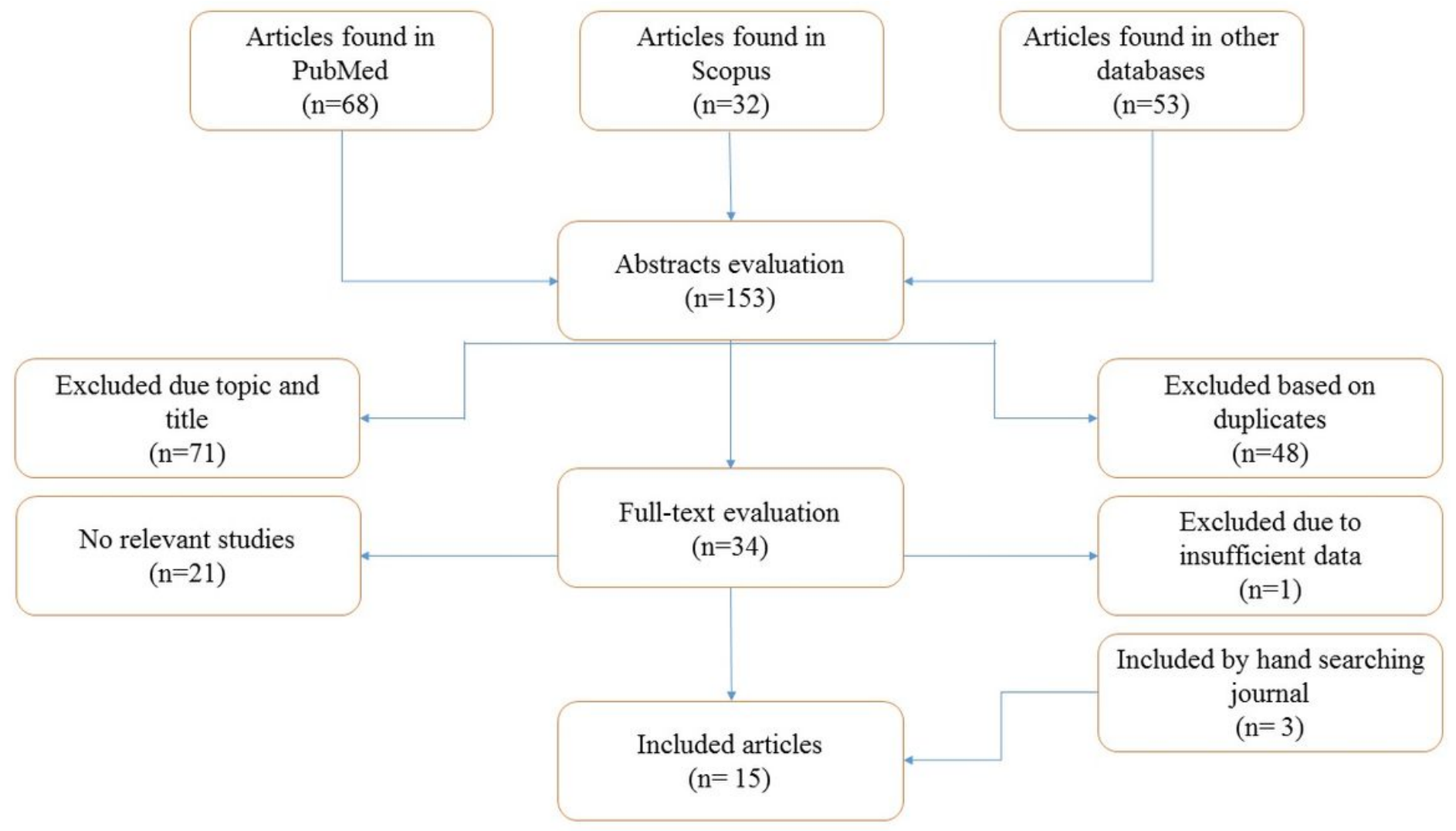

\section{Figure 1}

The flowchart of search strategy. 